\title{
Age and gender variations in the cone-beam computed tomographic location of mandibular canal: Implications for mandibular sagittal split osteotomy
}

\author{
Mohammad H. Al-Shayyab ${ }^{1}$, Khalid Qabba'ah ${ }^{2}$, Firas Alsoleihat ${ }^{3}$, Zaid H. Baqain ${ }^{4}$
}

\begin{abstract}
${ }^{1}$ MSc (Eng.), FFD RCS (Ire.). Assistant Dean, Associate Professor in Oral and Maxillofacial Surgery, Department of Oral and Maxillofacial Surgery, Oral Medicine and Periodontology, School of Dentistry, The University of Jordan, Amman, Jordan 11942 ${ }^{2}$ BDS. Resident in Oral and Maxillofacial Surgery, Department of Oral and Maxillofacial Surgery, Oral Medicine and Periodontology, School of Dentistry, The University of Jordan, Amman, Jordan 11942

${ }^{3}$ BDS, PhD. Assistant Dean. Associate Professor in the Department of Conservative Dentistry, Department of Conservative Dentistry, School of Dentistry, The University of Jordan, Amman, Jordan 11942

${ }^{4}$ MSc (Eng.), FDS RCS (Eng.). Vice president, Professor in Oral and Maxillofacial Surgery, Department of Oral and Maxillofacial Surgery, Oral Medicine and Periodontology, School of Dentistry, The University of Jordan, Amman, Jordan 11942
\end{abstract}

Correspondence:

Department of Oral and Maxillofacial Surgery

School of Dentistry, The University of Jordan

Amman, Jordan 11942

mshayyab@hotmail.com

Received: 08/01/2019 Accepted: 06/05/2019

\begin{abstract}
Al-Shayyab MH, Qabba'ah K, Alsoleihat F, Baqain ZH. Age and gender variations in the cone-beam computed tomographic location of mandibular canal: Implications for mandibular sagittal split osteotomy. Med Oral Patol Oral Cir Bucal. 2019 Jul 1;24 (4):e545-54.

http://www.medicinaoral.com/medoralfree01/v24i4/medoralv24i4p545.pdf
\end{abstract}

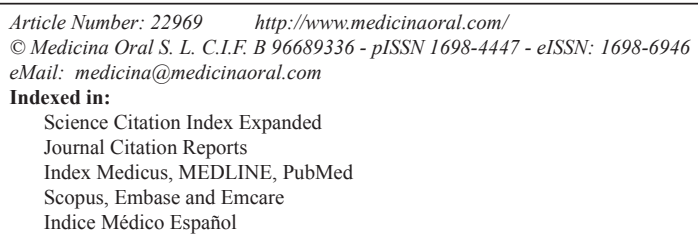

\begin{abstract}
Background: Mandibular sagittal split osteotomy (MSSO) may incur unfavorable split and sensorineural injuries. Knowledge of the anatomic location of the mandibular canal (MC) and bone thickness in the region of interest for MSSO, and the possible variations by age and gender can assist in avoiding such complications. Purpose: To study the location of the MC and bone thickness in the region of MSSO by cone-beam computed tomography (CBCT) radiographs and to evaluate the possible variations by age and gender in a Jordanian population.

Material and Methods: This retrospective radio-anatomical study examined all CBCT radiographs for patients treated over three years at the University of Jordan Hospital, Amman, Jordan. Distances from the MC to the cortical external surfaces and MC diameter (MCD) were measured by a reliable observer at three predetermined regions for MSSO: region (A) [mandibular foramen area], region (B) [mandibular angle area] and region (C) [directly mesial to the second molar]. Gender and age differences in all measurements were then compared using non-parametric Mann-Whitney U test.

Results: The final study radiographs comprised a total of 202 CBCT belonged to a cohort of 202 subjects; 91 males $(45.1 \%)$ and 111 (54.9\%) females, with mean age ( \pm SD) of $42.94 \pm 18.54$ years (range 18-90 years). Whereas only the bone thickness superior, buccal and inferior to MC in regions (B) and (C), and MCD in the three regions exhibited significant $(p<0.05)$ gender differences, all measured distances exhibited statistically significant $(p<$ $0.05)$ differences between young and adult patients.
\end{abstract}


Conclusion: The location of MC and bone thickness in the region of MSSO were significantly variable according to age, but exhibited sexual diamorphism only in regions (B) and (C). This fundamental knowledge should be considered during MSSO planning.

Key words: Cone-Beam Computed Tomography, mandibular canal, mandibular sagittal split osteotomy.

\section{Introduction}

Mandibular sagittal split osteotomy (MSSO) is the standard orthognathic surgical procedure commonly indicated for correction of mandibular deformities, such as prognathism, retrognathism and asymmetry (1). This technique was first popularized by Trauner and Obwegeser in 1957 (2). Since then, it had undergone several modifications aiming at minimizing the risk of important complications, such as unfavorable fractures (bad splits) and sensorineural disturbances in the lower lip, gingiva and chin region $(3,4)$. Such complications can negatively affect the recovery and daily life of patients submitted to orthognathic surgery (5), and seem to be clearly related to the positioning and depth of the osteotomy cuts during MSSO (4). Therefore, the osteotomy design should be decided based on the bone thickness and mandibular morphology in the vicinity of the inferior alveolar nerve (IAN) $(6,7)$.

Ample literature (8-11) investigated mandibular morphology using various anatomical and radiological methods, and reported variations possibly related to ethnicity, age, gender, dental status and dentofacial skeletal relationships. However, very few published English literature $(12,13)$ investigated the bone thickness and mandibular morphology in the vicinity of the IAN for MSSO using advanced imaging, and reported no significant variations among patients with different dentofacial skeletal relationships. To the authors' knowledge, no published English literature investigated such variations by both age and gender for MSSO. Hence, the aim of this study was to investigate, through cone-beam computed tomography (CBCT) radiographs, the anatomic location of the mandibular canal (MC) and bone thickness in the region of MSSO, and to demonstrate any possible variations by age and gender in a Jordanian population; it has been investigated for the first time in this population.

\section{Material and Methods}

-Study subjects

This retrospective radio-anatomical study reviewed all available CBCT radiographs at the University of Jordan Hospital (UJH), Amman, Jordan. The UJH is a referral centre located at the capital city of Amman and provides a comprehensive health service for more than half a million people annually. The Research Ethics Committee at UJH approved this study (reference number $10 / 2018 / 21807$ ), and the study was conducted in full ac- cordance with the Declaration of Helsinki. A total of $233 \mathrm{CBCT}$ radiographs primarily satisfied the inclusion criteria and belonged to 233 Jordanian subjects referred to and treated in the Department of Dentistry at the UJH for various dentofacial problems, between September 2015 and August 2018. Only CBCT radiographs showing an optimal viewing and diagnostic quality for patients aged $\geq 18$ years and presented with a complete set of mandibular posterior teeth and facial symmetry were included in this study. Those showing evidence of pathological lesions, missing or impacted teeth, or previous mandibular surgery as well as any developmental anomalies altering the position of the teeth, the $\mathrm{MC}$ or other concerned landmarks were excluded.

-CBCT radiographs

A senior radiology technician acquired all CBCT radiographs used in this study, according to the manufacturer's instructions and a strict, standardized scanning protocol at the UJH. These radiographs were obtained using a CBCT scanner (CS 9300. Carestream Health, Inc., 10622 AL 93 SS 0314, France, 2014), which was made at $60-90 \mathrm{kVp}$ and $2-15 \mathrm{~mA}$ at different resolutions, with an exposure time of $4-16 \mathrm{~s}$ and a voxel size of 90-300 $\mu \mathrm{m}$ depending on the field of view. Analysis of CBCT radiographs were also made at $2 \mathrm{~mm}$ slice thickness.

-Calibration and inter-observer reliability

To avoid inter-observer differences, all CBCT radiographs used in this study were evaluated by the same observer [a senior oral and maxillofacial surgery (OMFS) resident]. This observer was calibrated to identify the $\mathrm{MC}$ and other concerned landmarks in the region of MSSO using a set of CBCT radiographs not included in the study for the purpose of training. Calibration sessions were presented to the observer by the senior maxillofacial radiologist at the UJH and entailed practical demonstrations and discussions on the identifications of $\mathrm{MC}$ and other concerned landmarks and on the method of using the CBCT software to measure distances between these landmarks. This stage was considered successfully completed when the observer demonstrated a proficient capability of identifying the $\mathrm{MC}$ and other concerned landmarks and of measuring distances between these landmarks. The observer was then given a set of CBCT radiographs not included in the study to measure all required distances, which were re-measured by the maxillofacial radiologist. The interobserver reliability was then assessed using the intra- 
class correlation coefficient (ICC), which was overall ranged from 83.9 to $99.8 \%$, with $95 \%$ confidence interval (CI) of 0.592-0.999 for all various measurements. This indicated an excellent level of agreement $(P<$ 0.001 ) between the observer and the maxillofacial radiologist, and would suggest that the observer was reliable in examining the experimental $\mathrm{CBCT}$ radiographs.

-CBCT analysis and evaluation techniques

When conducting $\mathrm{CBCT}$ analysis, the calibrated observer was blinded to any other patients' details. All CBCT radiographs were examined in standard viewing conditions by enhancing the software's image processing tool for adjusting the brightness and contrast values to ensure optimal visualization. In addition, the software allowed the axial, cross-sectional, sagittal, and panoramic reconstructions, which were pre-oriented and used as necessary to identify the location of the MC and other concerned landmarks. For each patient, the cross-sectional scan was taken and valid measurements were ensured by obtaining these measurements through the same reproducible lines at the level of three specific regions of interest for MSSO (Fig. 1), as recently described (7):

- Region (A): the mandibular foramen (MF) area (the first view in which the foramen was detected).

- Region (B): the transitional area between the mandibular ramus and body (mandibular angle area) [obtained through a straight line that was crossing the $\mathrm{MC}$ and tapping the most anterior border of the ramus].

- Region $(\mathrm{C})$ : the area directly mesial to the second mandibular molar.

In the reconstructed cross-sectional views (Fig. 2), the software ruler was enhanced to measure distances (in millimeters) between the $\mathrm{MC}$ and specific mandibular landmarks used in recent studies $(7,11,12)$ : in region (A), the distance between the superior cortex of MF and the fusion between the buccal and lingual cortices (FBLC) above MF [MC-FBLC], and between the outer surface of MC and the external surface of the buccal (B) cortical plate [MC-B]; in regions (B) and (C), distances between the outer surface of $\mathrm{MC}$ and the external surface of the superior (S) [MC-S], lingual (L) [MC-L], inferior (I) $[\mathrm{MC}-\mathrm{I}]$ and $\mathrm{B}[\mathrm{MC}-\mathrm{B}]$ cortical plates of mandibular bone; and in the three regions, the largest diameter of the MC [MCD] (Fig. 2).

All measurements for each subject were assessed on the right and left sides, and hence, $466 \mathrm{CBCT}$ radiographs of the $\mathrm{MC}$ and of the bone thickness between the $\mathrm{MC}$ and other concerned landmarks were primarily analyzed. Patients' file number, age, and gender were then recorded, and according to their age, they were divided into two groups; young (18-40 years), and adult ( $>40$ years). This classification border considered the fact, demonstrated in a recent study (8) on the same popu-

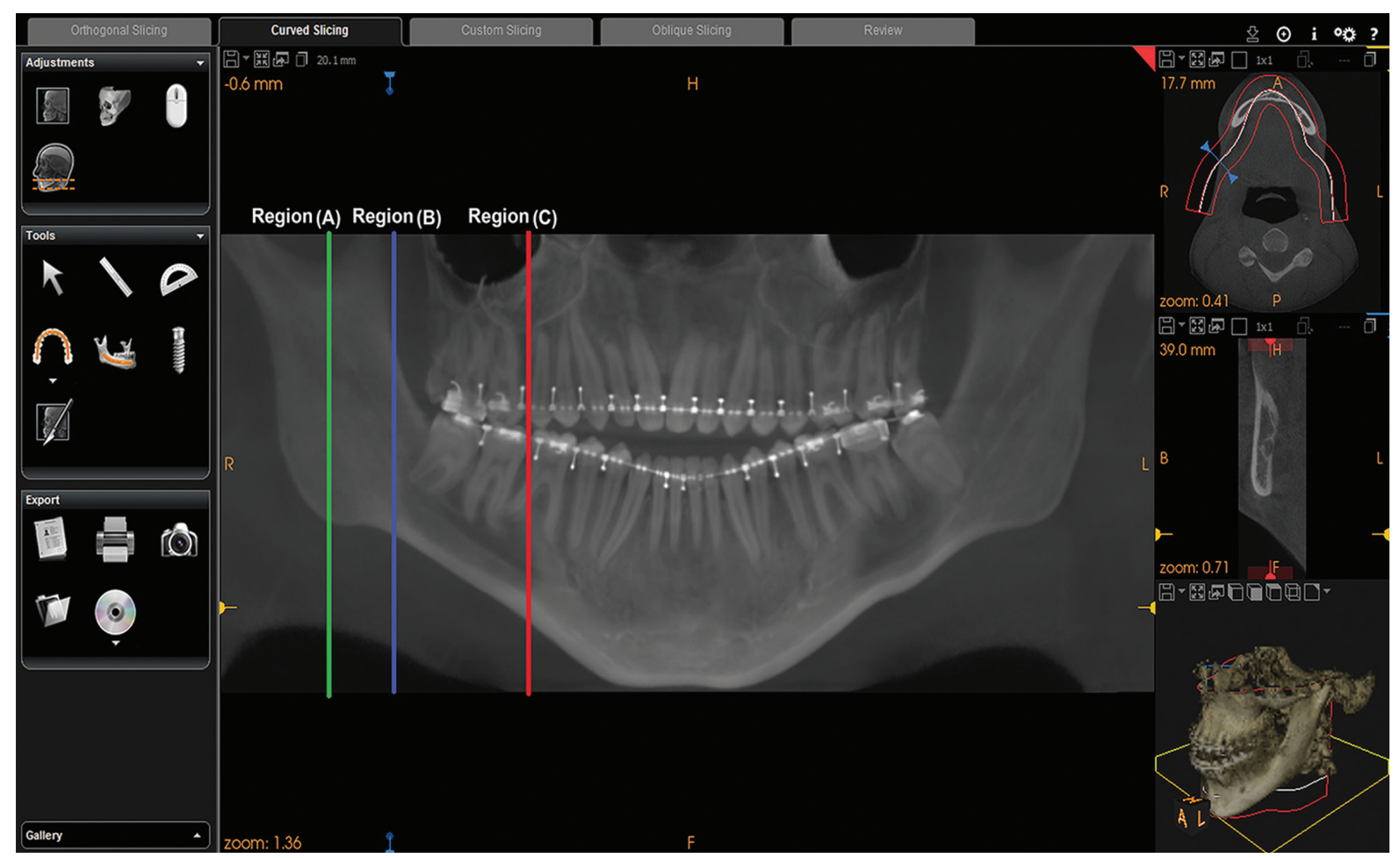

Fig. 1: Reconstructed panoramic view showing the lines at which all measurements were obtained at the level of three specific regions of interest for mandibular sagittal split osteotomy. Region (A): The mandibular foramen (MF) area; Region (B): The transitional area between the mandibular ramus and body; Region (C): The area directly mesial to the second mandibular molar. 


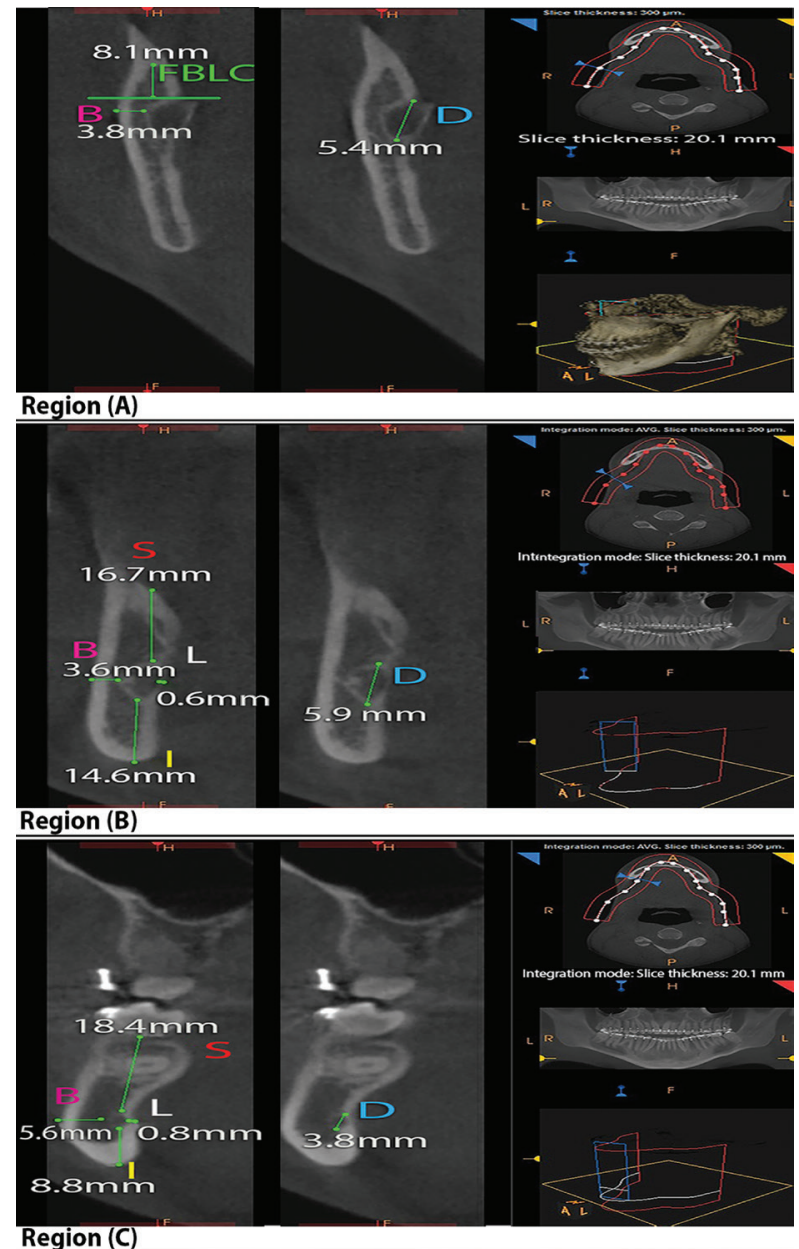

Fig. 2: Reconstructed cross-sectional views with the software ruler measuring distances from the external surface of mandibular canal (MC) to the cortical external surfaces. Region (A): The mandibular foramen (MF) area; Region (B): The transitional area between the mandibular ramus and body; Region (C): The area directly mesial to the second mandibular molar; FBLC: Fusion between the buccal and lingual cortices above MF; S: The external surface of the alveolar crest; B: The external surface of the buccal cortical plate; I: The external surface of the inferior border of the mandible; L: The external surface of the lingual cortical plate; D: Mandibular canal diameter.

lation, that mandibular growth and remodeling in the vicinity of the IAN goes on until the bone has reached the adult size (40 years), following which the deposition and resorption process is imbalanced and results in a significantly decreased ramus measurements. It would also create comparable cohorts in this study.

-Intra-observer reliability and power analysis

The same calibrated observer was asked to measure all distances in the included CBCT images twice in a twomonth interval. The ICC was then used to assess the level of intra-observer agreement between the two measurement sessions, and hence intra-observer reliability. The statistical software package $G *$ Power version 3.1.5 (Franz Faul, Universität Kiel, Kiel, Germany, 1992) was used to calculate the statistical power of this study.
This was performed retrospectively, and hence post-hoc power analysis was conducted and a Mann-Whitney $U$ test of two independent groups was set as a statistical test to perform power analyses for age and gender comparisons at an effect size of 0.5 (Cohen's medium effect size), $\alpha$ error probability of 0.05 , and a sample size of 202 subjects valid for age and gender analyses; 93 young subjects versus 109 adults, and 91 males versus 111 females.

\section{-Statistical analysis}

The Statistical Package for Social Sciences for Windows version 19 (SPSS, Chicago, IL, USA) was used to analyze the collected data, and the statistical significance was based on probability values of $<0.05$. Descriptive statistics were produced for the included subjects and their measurements, overall and within the groups. To obtain valid results, data was ensured to pass the assumptions required for parametric and non-parametric tests. The Shapiro-Wilk test was initially used to assess the normality of the collected data and indicated nonparametric tests to compare mean ranks, overall and within the groups. As such, differences in the measurements between the right and left hemi-mandibles were analyzed using Wilcoxon signed-ranks test. Gender and age differences in all measurements were also compared using Mann-Whitney U test, overall and within the same group.

\section{Results}

\section{-Study subjects}

Out of the 233 CBCT radiographs primarily satisfied the inclusion criteria for this study, 31 were further excluded; seven belonged to subjects aged $<18$ years, eight showed poor diagnostic quality and mandibular asymmetry and 16 showed evidence of pathological lesions, missing or impacted teeth, or previous mandibular surgery as well as developmental disturbances altering mandibular morphology. The final study radiographs comprised a total of 202 CBCT belonged to a cohort of 202 subjects; 91 males (45.1\%) and 111 (54.9\%) females, with mean age $( \pm \mathrm{SD})$ of $42.94 \pm 18.54$ years (range 18-90 years). Descriptive statistics of the final study cohort are presented in Table 1, overall and within the same gender and age group.

-Intra-examiner reliability and power analysis

Repeated CBCT measurements by the same reliable observer indicated a significant $(P<0.001)$ level of intra-observer agreement between the two measurement sessions; an overall ICC ranging from $95.5 \%$ to $99.4 \%$, with $95 \%$ confidence interval (CI) of $0.941-0.995$ for all various measurements (Table 2). Therefore, the intraobserver difference was random and statistically not significant. Analysis computation for this study also yielded $97.0 \%$ statistical power for age and gender analyses; thus, demonstrating that such retrospective study 
Table 1: Descriptive statistics of the study groups (age and sex) $(\mathrm{N}=202)$.

\begin{tabular}{|l|c|c|c|c|c|}
\hline \multirow{2}{*}{ Age group } & \multirow{N}{*}{$\mathbf{N}$} & Male & Female & \multicolumn{2}{|c|}{ Age, years } \\
\cline { 3 - 6 } & & $\mathbf{n}(\%)$ & $\mathbf{n}(\%)$ & Mean \pm SD & Range \\
\hline Young & 93 & $35(37.6)$ & $58(62.4)$ & $25.8 \pm 7.1$ & $18-40$ \\
\hline Adult & 109 & $56(51.4)$ & $53(48.6)$ & $57.6 \pm 11.4$ & $41-90$ \\
\hline Total & 202 & $91(45.1)$ & $111(55.9)$ & $42.9 \pm 18.5$ & $18-90$ \\
\hline
\end{tabular}

SD: Standard deviation.

Table 2: Intra-observer reliability.

\begin{tabular}{|c|c|c|c|c|c|}
\hline \multirow[t]{2}{*}{ Variable } & \multirow[t]{2}{*}{ Region } & \multirow{2}{*}{$\begin{array}{l}\text { Intraclass Correlation } \\
\text { Coefficient (ICC) }\end{array}$} & \multicolumn{2}{|c|}{$95 \%$ confidence interval } & \multirow[t]{2}{*}{$P$ value* } \\
\hline & & & Lower bound & Upper bound & \\
\hline MC-FBLC & (A) & 0.993 & 0.991 & 0.995 & $<0.001$ \\
\hline \multirow[t]{2}{*}{ MC-S } & (B) & 0.993 & 0.991 & 0.995 & $<0.001$ \\
\hline & (C) & 0.994 & 0.992 & 0.995 & $<0.001$ \\
\hline \multirow[t]{3}{*}{ MC-B } & (A) & 0.968 & 0.958 & 0.976 & $<0.001$ \\
\hline & (B) & 0.978 & 0.971 & 0.983 & $<0.001$ \\
\hline & (C) & 0.979 & 0.972 & 0.984 & $<0.001$ \\
\hline \multirow[t]{2}{*}{ MC-I } & (B) & 0.993 & 0.990 & 0.995 & $<0.001$ \\
\hline & (C) & 0.992 & 0.990 & 0.994 & $<0.001$ \\
\hline \multirow[t]{2}{*}{ MC-L } & (B) & 0.977 & 0.970 & 0.983 & $<0.001$ \\
\hline & (C) & 0.976 & 0.968 & 0.982 & $<0.001$ \\
\hline \multirow[t]{3}{*}{ MCD } & (A) & 0.955 & 0.941 & 0.966 & $<0.001$ \\
\hline & (B) & 0.958 & 0.945 & 0.968 & $<0.001$ \\
\hline & (C) & 0.957 & 0.971 & 0.984 & $<0.001$ \\
\hline
\end{tabular}

Region (A): The mandibular foramen area; Region (B): The transitional area between the mandibular ramus and body; Region (C): The area directly mesial to the second mandibular molar; MC: The outer surface of the mandibular canal; FBLC: Fusion between the buccal and lingual cortices above mandibular foramen; S, The external surface of the alveolar crest; B: The external surface of the buccal cortical plate; I: The external surface of the inferior border of the mandible; L: The external surface of the lingual cortical plate; MCD: Mandibular canal diameter; $P$ value*: $P$-value of Intraclass Correlation Coefficient (ICC).

was very adequate for detecting age and gender statistical variations.

-Comparison of measurements between the right and left hemi-mandibles

In the three regions of interest for BSSO, there were no statistical significant $(P>0.05)$ differences between measurements in the right and left hemi-mandibles. Therefore, measurements from both sides have been averaged and then used in later analyses.

-Comparison of the distances between males and females

The average, minimum and maximum measurements and standard deviation among males and females are presented in Table 3, overall and within the same age group. Whereas the MC-FBLC, MC-B in region (A) and MC-L distances were not significantly $(P>0.05)$ different between males and females, the MC-S in region (C) and $\mathrm{MCD}$ in the three regions were significantly longer in males than in females, overall [MC-S (region $\mathrm{C}$ ): $\mathrm{U}=$ 3505, $P<0.001$; MCD: $\mathrm{U}=3514.5-3532.5, P<0.001]$ and within the same age group [young $\mathrm{MC}-\mathrm{S}$ (region $\mathrm{C}$ ): $\mathrm{U}=476.0, P<0.001$; adult MC-S (region $\mathrm{C}$ ): $\mathrm{U}=1109.0$, $P<0.05$; young MCD: $\mathrm{U}=597.5-619.5, P=0.001-0.002$; adult MCD: $\mathrm{U}=919.5-923.5, P=0.001]$. However, The average MC-S and MC-I in region (B), and MC-B in regions (B) and (C) distances exhibited significant higher measurements in males than in females within a specific age group [young MC-S (region B): $\mathrm{U}=561, P<$ 0.001; adult MC-I (region $\mathrm{B}$ ): $\mathrm{U}=855.0, P<0.001$; adult MC-B (region B): $\mathrm{U}=952.0, P<0.05$; adult MC-B (region $\mathrm{C}$ ): $\mathrm{U}=744.5, P<0.001]$. By contrast, the average MC-I distance in region (C) exhibited significant higher measurements in females than in males only among the young age group $(\mathrm{U}=310.0, P<0.001)$ (Table 3$)$.

-Comparison of the distances between young and adult patients

The average, minimum and maximum measurements and standard deviation among young and adult age groups are presented within the same gender in Table 3, and overall in Table 4; adults exhibited a decrease in the 
Table 3: Comparison of the average distances at the level of three specific regions of interest for mandibular sagittal split osteotomy (BSSO); between the two age groups within the same gender, and between males and females, overall and within the same age group ( $N=202)$.

\begin{tabular}{|c|c|c|c|c|c|c|c|}
\hline \multirow{2}{*}{ 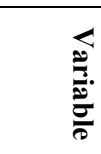 } & \multirow[b]{2}{*}{ 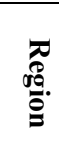 } & \multirow{2}{*}{$\begin{array}{c}\text { Age } \\
\text { group }\end{array}$} & \multicolumn{5}{|c|}{ Gender } \\
\hline & & & $\begin{array}{c}\text { Male } \\
n(\text { mean } \pm S D)(\min -\mathrm{max})\end{array}$ & $P^{*}$ & $\begin{array}{c}\text { Female } \\
n(\text { mean } \pm S D)(\text { min-max })\end{array}$ & $P^{*}$ & $P * *$ \\
\hline \multirow{3}{*}{$\begin{array}{l}\text { MC- } \\
\text { FBLC }\end{array}$} & (A) & Young & $35(10.7 \pm 1.6)(5.0-15.2)$ & $<0.001$ & $58(10.2 \pm 1.8)(5.1-15.5)$ & $<0.001$ & 0.054 \\
\hline & & Adult & $56(8.6 \pm 2.3)(4.4-14.7)$ & & $53(8.3 \pm 2.1)(4.4-14.3)$ & & 0.409 \\
\hline & & Total & $91(9.4 \pm 2.3)(4.4-15.2)$ & & $111(9.3 \pm 2.2)(4.4-15.5)$ & & 0.564 \\
\hline \multirow[t]{6}{*}{ MC-S } & (B) & Young & $35(19.0 \pm 2.8)(12.0-26.0)$ & $<0.001$ & $58(17.2 \pm 2.3)(10.0-25.1)$ & 0.048 & $<0.001$ \\
\hline & & Adult & $56(16.4 \pm 1.7)(10.9-18.4)$ & & $53(16.6 \pm 1.5)(12.0-22.0)$ & & 0.380 \\
\hline & & Total & $91(17.4 \pm 2.5)(10.9-26.0)$ & & $111(16.9 \pm 2.0)(10.0-25.1)$ & & 0.018 \\
\hline & (C) & Young & $35(19.2 \pm 2.8)(12.2-26.2)$ & $<0.001$ & $58(17.1 \pm 2.2)(10.2-25.0)$ & 0.041 & $<0.001$ \\
\hline & & Adult & $56(16.6 \pm 1.7)(11.1-18.6)$ & & $53(16.4 \pm 1.6)(11.9-21.9)$ & & 0.023 \\
\hline & & Total & $91(17.6 \pm 2.5)(11.1-26.2)$ & & $111(16.7 \pm 2.0)(10.2-25.0)$ & & $<0.001$ \\
\hline \multirow[t]{9}{*}{ MC-B } & (A) & Young & $35(2.8 \pm 1.1)(0.7-5.1)$ & $<0.001$ & $58(3.1 \pm 1.0)(0.7-5.3)$ & $<0.001$ & 0.510 \\
\hline & & Adult & $56(3.9 \pm 0.6)(3.2-5.9)$ & & $53(3.8 \pm 0.8)(1.9-6.1)$ & & 0.253 \\
\hline & & Total & $91(3.5 \pm 1.0)(0.7-5.9)$ & & $111(3.4 \pm 1.0)(0.7-6.1)$ & & 0.141 \\
\hline & (B) & Young & $35(4.0 \pm 1.4)(1.6-8.9)$ & $<0.001$ & $58(4.2 \pm 1.3)(1.9-9.5)$ & 0.004 & 0.517 \\
\hline & & Adult & $56(5.1 \pm 1.0)(4.2-9.2)$ & & $53(4.7 \pm 1.0)(2.1-8.7)$ & & 0.001 \\
\hline & & Total & $91(4.6 \pm 1.2)(1.6-9.2)$ & & $111(4.4 \pm 1.2)(1.9-9.5)$ & & 0.009 \\
\hline & (C) & Young & $35(5.1 \pm 1.4)(2.6-10.1)$ & $<0.001$ & $58(5.4 \pm 1.5)(1.9-10.5)$ & 0.022 & 0.543 \\
\hline & & Adult & $56(6.4 \pm 1.0)(5.4-9.9)$ & & $53(5.8 \pm 1.0)(2.9-9.6)$ & & $<0.001$ \\
\hline & & Total & $91(5.9 \pm 1.3)(2.6-10.1)$ & & $111(5.6 \pm 1.3)(1.9-10.5)$ & & 0.001 \\
\hline \multirow[t]{6}{*}{ MC-I } & (B) & Young & $35(9.1 \pm 2.6)(4.0-16.0)$ & $<0.001$ & $58(9.7 \pm 2.2)(3.8-15.0)$ & 0.034 & 0.236 \\
\hline & & Adult & $56(11.6 \pm 1.7)(9.6-16.6)$ & & $53(10.4 \pm 1.5)(5.0-15.0)$ & & $<0.001$ \\
\hline & & Total & $91(10.6 \pm 2.4)(4.0-16.6)$ & & $111(10.1 \pm 1.9)(3.8-15.0)$ & & 0.009 \\
\hline & (C) & Young & $35(7.7 \pm 2.3)(3.8-14.5)$ & 0.049 & $58(10.1 \pm 2.1)(3.5-16.1)$ & 0.001 & $<0.001$ \\
\hline & & Adult & $56(8.6 \pm 1.9)(3.5-13.7)$ & & $53(9.1 \pm 1.5)(3.7-13.7)$ & & 0.069 \\
\hline & & Total & $91(8.3 \pm 2.1)(3.5-14.5)$ & & $111(9.6 \pm 1.9)(3.5-16.1)$ & & $<0.001$ \\
\hline \multirow[t]{6}{*}{ MC-L } & (B) & Young & $35(3.0 \pm 1.0)(1.2-6.8)$ & $<0.001$ & $58(3.1 \pm 0.9)(1.4-6.8)$ & $<0.001$ & 0.703 \\
\hline & & Adult & $56(2.5 \pm 1.2)(0.6-7.3)$ & & $53(2.7 \pm 1.4)(0.7-7.3)$ & & 0.357 \\
\hline & & Total & $91(2.7 \pm 1.2)(0.6-7.3)$ & & $111(2.9 \pm 1.2)(0.7-7.3)$ & & 0.061 \\
\hline & (C) & Young & $35(2.8 \pm 1.0)(0.8-6.4)$ & $<0.001$ & $58(2.9 \pm 0.9)(1.2-6.6)$ & $<0.001$ & 0.858 \\
\hline & & Adult & $56(2.3 \pm 1.2)(0.8-7.1)$ & & $53(2.5 \pm 1.4)(0.8-7.1)$ & & 0.293 \\
\hline & & Total & $91(2.5 \pm 1.2)(0.8-7.1)$ & & $111(2.7 \pm 1.2)(0.8-7.1)$ & & 0.064 \\
\hline \multirow[t]{9}{*}{ MCD } & (A) & Young & $35(3.2 \pm 0.7)(1.9-5.3)$ & 0.003 & $58(2.9 \pm 0.7)(1.5-5.2)$ & 0.001 & 0.002 \\
\hline & & Adult & $56(2.9 \pm 0.8)(1.9-5.5)$ & & $53(2.5 \pm 0.9)(1.5-5.3)$ & & 0.001 \\
\hline & & Total & $91(3.0 \pm 0.8)(1.9-5.5)$ & & $111(2.7 \pm 0.9)(1.5-5.3)$ & & $<0.001$ \\
\hline & (B) & Young & $35(4.2 \pm 0.8)(2.1-6.3)$ & 0.002 & $58(3.9 \pm 0.8)(2.0-6.2)$ & 0.001 & 0.001 \\
\hline & & Adult & $56(3.9 \pm 0.8)(2.9-6.5)$ & & $53(3.5 \pm 0.9)(2.5-6.3)$ & & 0.001 \\
\hline & & Total & $91(4.0 \pm 0.8)(2.1-6.5)$ & & $111(3.7 \pm 0.9)(2.0-6.3)$ & & $<0.001$ \\
\hline & (C) & Young & $35(3.3 \pm 0.7)(2.0-5.4)$ & 0.003 & $58(3.0 \pm 0.7)(1.6-5.3)$ & 0.001 & 0.002 \\
\hline & & Adult & $56(3.0 \pm 0.8)(2.1-5.7)$ & & $53(2.6 \pm 0.9)(1.7-5.4)$ & & 0.001 \\
\hline & & Total & $91(3.1 \pm 0.8)(2.0-5.7)$ & & $111(2.8 \pm 0.8)(1.6-5.4)$ & & $<0.001$ \\
\hline
\end{tabular}

SD: Standard deviation; min: Minimum; max: Maximum; Region (A): The mandibular foramen area; Region (B): The transitional area between the mandibular ramus and body; Region $(\mathrm{C})$ : The area directly mesial to the second mandibular molar; MC: The outer surface of the mandibular canal; FBLC: Fusion between the buccal and lingual cortices above mandibular foramen; S: The external surface of the alveolar crest; B: The external surface of the buccal cortical plate; I: The external surface of the inferior border of the mandible; L: The external surface of the lingual cortical plate; MCD: Mandibular canal diameter; $P^{*}: P$-value of Mann-Whitney U test (significance between the two age groups); $P^{* *}: P$-value of Mann-Whitney $\mathrm{U}$ test (significance of male to female). 
Table 4: Comparison of the average distances at the level of three specific regions of interest for mandibular sagittal split osteotomy (BSSO); between the two age groups in the entire cohort $(\mathrm{N}=202)$.

\begin{tabular}{|c|c|c|c|c|}
\hline Variable & Region & Age group & $n(m e a n \pm S D)(\min -m a x)$ & $P^{*}$ \\
\hline \multirow[t]{3}{*}{ MC-FBLC } & (A) & Young & $93(10.4 \pm 1.8)(5.0-15.5)$ & $<0.001$ \\
\hline & & Adult & $109(8.5 \pm 2.2)(4.4-14.7)$ & \\
\hline & & Total & $202(9.4 \pm 2.2)(4.4-15.5)$ & \\
\hline \multirow[t]{6}{*}{ MC-S } & (B) & Young & $93(17.9 \pm 2.6)(10.0 \pm 26.0)$ & $<0.001$ \\
\hline & & Adult & $109(16.5 \pm 1.6)(10.9-22.0)$ & \\
\hline & & Total & $202(17.1 \pm 2.2)(10.0-26.0)$ & \\
\hline & (C) & Young & $93(17.9 \pm 2.7)(10.2-26.2)$ & $<0.001$ \\
\hline & & Adult & $109(16.5 \pm 1.7)(11.1-21.9)$ & \\
\hline & & Total & $202(17.1 \pm 2.3)(10.2-26.2)$ & \\
\hline \multirow[t]{9}{*}{ MC-B } & (A) & Young & $93(3.0 \pm 1.0)(1.7-5.3)$ & $<0.001$ \\
\hline & & Adult & $109(3.9 \pm 1.7)(1.9-6.1)$ & \\
\hline & & Total & $202(3.5 \pm 1.0)(1.7-6.1)$ & \\
\hline & (B) & Young & $93(4.1 \pm 1.3)(1.6-9.5)$ & $<0.001$ \\
\hline & & Adult & $109(4.9 \pm 1.0)(2.1-9.2)$ & \\
\hline & & Total & $202(4.5 \pm 1.2)(1.6-9.5)$ & \\
\hline & (C) & Young & $93(5.3 \pm 1.4)(1.9-10.5)$ & $<0.001$ \\
\hline & & Adult & $109(6.1 \pm 1.0)(2.9-9.9)$ & \\
\hline & & Total & $202(5.7 \pm 1.3)(1.9-10.5)$ & \\
\hline \multirow[t]{6}{*}{ MC-I } & (B) & Young & $93(9.5 \pm 2.3)(3.8-16.0)$ & $<0.001$ \\
\hline & & Adult & $109(11.0 \pm 1.7)(5.0-16.6)$ & \\
\hline & & Total & $202(10.3 \pm 2.1)(3.8-16.6)$ & \\
\hline & (C) & Young & $93(9.2 \pm 2.4)(3.5-16.1)$ & 0.049 \\
\hline & & Adult & $109(8.9 \pm 1.7)(3.5-13.7)$ & \\
\hline & & Total & $202(9.0 \pm 2.1)(3.5-16.1)$ & \\
\hline \multirow[t]{6}{*}{ MC-L } & (B) & Young & $93(3.1 \pm 0.9)(1.2-6.8)$ & $<0.001$ \\
\hline & & Adult & $109(2.6 \pm 1.3)(0.6-7.3)$ & \\
\hline & & Total & $202(2.8 \pm 1.2)(0.6-7.30$ & \\
\hline & (C) & Young & $93(2.9 \pm 0.9)(.8-6.6)$ & $<0.001$ \\
\hline & & Adult & $109(2.4 \pm 1.3)(.8-7.1)$ & \\
\hline & & Total & $202(2.6 \pm 1.2)(.8-7.1)$ & \\
\hline \multirow[t]{9}{*}{ MCD } & (A) & Young & $93(3.0 \pm 0.7)(1.5-5.3)$ & 0.003 \\
\hline & & Adult & $109(2.7 \pm 0.9)(1.5-5.5)$ & \\
\hline & & Total & $202(2.8 \pm 0.8)(1.5-5.5)$ & \\
\hline & (B) & Young & $93(4.0 \pm 0.8)(2.0-6.3)$ & 0.002 \\
\hline & & Adult & $109(3.7 \pm 0.9)(2.5-6.5)$ & \\
\hline & & Total & $202(3.8 \pm 0.8)(2.0-6.5)$ & \\
\hline & (C) & Young & $93(3.1 \pm 0.7)(1.6-5.4)$ & 0.003 \\
\hline & & Adult & $109(2.8 \pm 0.9)(1.7-5.7)$ & \\
\hline & & Total & $202(2.9 \pm 0.8)(1.6-5.7)$ & \\
\hline
\end{tabular}

SD: Standard deviation; min: Minimum; max: Maximum; Region (A): The mandibular foramen area; Region (B): The transitional area between the mandibular ramus and body; Region $(\mathrm{C})$ : The area directly mesial to the second mandibular molar; MC: The outer surface of the mandibular canal; FBLC: Fusion between the buccal and lingual cortices above mandibular foramen; S: The external surface of the alveolar crest; B: The external surface of the buccal cortical plate; I: The external surface of the inferior border of the mandible; L: The external surface of the lingual cortical plate; MCD: Mandibular canal diameter; P*: P-value of Mann-Whitney U test (significance between the two age groups). 
average values of MC-FBLC, MC-S, MC-L, and MCD, and an increase in the average values of MC-B and MCI, compared with young patients. Mann-Whitney U test generally indicated that such age differences in these average distances were statistically significant, within the same gender (male: $\mathrm{U}=294.5-738.5, P=0.000-0.049$; female: $\mathrm{U}=621.5-1205.5, P=0.000-0.048$ ) (Table 3), and overall ( $\mathrm{U}=2067.0-4294.0, P=0.000-0.049)$ (Table 4).

\section{Discussion}

Knowledge of the anatomic location of the MC and bone thickness in the region of interest for MSSO and the possible variations by age and gender can help oral and maxillofacial surgeons to decide on the safest osteotomy design that is tailored to specific age and sex $(7,11,14)$, and may assist in evaluating the risk of bad split and the degree and extent of IAN injury resulting from both direct and indirect surgical trauma (15). CBCT is well suited for anatomic structures in the maxillofacial region $(16,17)$, and has several advantages over conventional CT and plain films: reduced effective radiation dose, fewer artifacts, and high accuracy and reproducibility (18).

In this study, the MC-FBLC, MC-B and MCD distances measured in region (A) are of clinical relevance to the horizontal osteotomy cut in MSSO, whereas the MC-B, MC-S, MC-L, MC-I and MCD distances measured in regions (B) and (C) are of clinical relevance to the sagittal and vertical osteotomies of MSSO. In this study, the $\mathrm{MC}$ generally presented a variable diameter that increased in region (B) and decreased in region (C) to become close to diameter in region (A), with a significantly longer diameter among adults and males when compared to young patients and females, respectively. The FBLC was significantly located at a mean height of $10.4 \mathrm{~mm}$ with a minimum distance of $5 \mathrm{~mm}$ in young patients compared with a mean height of $8.5 \mathrm{~mm}$ with a minimum distance of $4.4 \mathrm{~mm}$ in adults from the MF, with no significant sexual dimorphism. In addition, the MC-B distance in region (A) significantly measured an average distance of $3 \mathrm{~mm}$ in young patients compared with an average distance of $3.9 \mathrm{~mm}$ in adults, regardless of the gender. Interestingly, the sum values of the vertical and horizontal distances in regions (B) and (C) showed that female mandibles had smaller vertical dimension in region (B) and thinner buccolingual dimension in region (C) than males, but adult mandibles had a smaller vertical dimension with a buccolingual dimension similar to young mandibles. These results are a bit higher but comparable to the CBCT findings of Sekerci and Sahman (11) in a Turkish population and Scomparion et al. (7) in a Brazilin popula $\neg$ tion (as expected due to ancestral similarity), and to the findings of $\mathrm{Yu}$ and Wong (10) in a Taiwanese population (although unexpected due to ancestral dissimilarity) using
3DCT images; nevertheless the authors did not present any data regarding the comparison of the measurements in terms of age, but presented gender differences that were generally not in corroboration with this study. Noleto et al. (13) and Huang and Liao (12) demonstrated no significant variations in Brazilian and Chinese patients, respectively, with different dentofacial skeletal relationships, but reported nearly close measurements (as expected due to ancestral similarity to Brazilians, but unexpected due to ancestral dissimilarity to Chinese); however, the authors did not present any data regarding the comparison of the measurements in terms of age and sex and used the lingula, not the superior cortex of the MF, as a landmark for the measurements what can difficult the comparison with this study.

The findings of this study indicated an absolute positional bilateral symmetry of the MC and bone thickness in the region of MSSO, and this is in agreement with previous studies $(7,8,11)$. As for gender, gender did not always lead to a significant variation in distances measured in this study, and this is in agreement with recent studies $(7,11)$ and with the fact that genetic and environmental factors may also influence bone size and thickness $(8,19)$. However, measurements recorded in this study were significantly variable according to age and in concordance with the fact that the position of the MC in relation to other landmarks is not constant and affected by imbalanced growth and remodeling occurred with advancing age, characterized by thickening of the buccal and inferior aspects of mandibular bone and resorption of the superior and lingual aspects moving the $\mathrm{MC}$ in a superior and lingual direction and leaving a decreased amount of cancellous bone, and shorter ramus and mandibular posterior region $(8,20,21)$. Aarabi et al. (22) reported that patients with smaller amount of bone in region (A) and shorter rami were more likely to present bad splits in the lingual side of the distal segment during MSSO. A shorter ramus probably leads to a more difficult surgical access and shorter MC-FBLC distance. In addition, the smaller amount of bone in region (A) may contribute to mandible brittleness towards the forces applied during horizontal osteotomy (22). Aarabi et al. (22) also reported that patients with a thinner buccolingual distance in regions (B) and (C) presented an increased risk for unfavorable fractures in the distal or proximal segments during MSSO. Thus, the suggestion $(6,10,15)$ that the area between the first and second molars along the crest of the external oblique line is the best location to perform the vertical anterior osteotomy; presumably because of a greater buccal bone thickness, and hence a lower chance of IAN injury and badsplits. In addition, the shorter mandibular posterior region presents more difficult surgical access and may contribute to mandible brittleness towards the forces applied during sagittal and vertical osteotomies 
(24). Witherow et al., (23) in a study using panoramic radiographs, stated that patients with a mandibular retromolar height of $<2 \mathrm{~cm}$ and with a distance between the apices of the last molar tooth and the inferior border of the mandible of $<0.6 \mathrm{~cm}$ were at higher risk of developing unfavorable fractures of the lingual plate during MSSO. Furthermore, CBCT studies have shown that the distance between the mandibular canal and the split surface is correlated with the trigeminal somatosensory-evoked potential latency recovery (24). Thus, in light of the aforementioned literature $(6,8,10,15,20$ 23 ), the findings of the current study indicated a significantly increased chance of IAN injury and unfavorable fractures during MSSO among adults compared with young patients, with a slightly increased chance among females during sagittal and vertical osteotomies of MSSO. Therefore, the current study would suggest that the horizontal mandibular ramus osteotomy has to be performed at an average point not exceeding $9 \mathrm{~mm}$ in young patients and $7 \mathrm{~mm}$ in adults from the MF, and carried to the depth of the medial surface of the buccal cortex, which would be deeper in young patient than in adults; this would help the surgeon in avoiding a region that could considerably increase the incidence of unfavorable fractures (23). In addition, the sagittal mandibular osteotomy has to be performed more buccal and not deeper in adults and females than in young and female patients; this would help the surgeon in avoiding the IAN and providing adequate lingual bone thickness that could considerably decrease the incidence of unfavorable fractures in the distal or proximal segments (23). Furthermore, it is important to consider using miniplates and monocortical fixation in patients with thinner superior and lingual bone thickness, to avoid the transmission of forces to the lingual plate reducing the risk of unfavorable fractures (19). Our findings also suggest that the vertical mandibular osteotomy has to be carried to the depth of the medial surface of the buccal and inferior mandibular cortices, which would be deeper for the buccal osteotomy in adult and male patients, and for the inferior border osteotomy in adult and female patients; this would make the surgeon reach a depth that could considerably decrease the incidence of bad split and IAN injury during MSSO (15).

Despite the abovementioned findings, this research is not without limitations; it did not account for the possible variations by dentofacial skeletal relationships: although reported $(12,13)$ to be not significant, but should not be ignored in future research to achieve a reliable conclusion. In addition, differences in population and/ or measurement techniques could be the reason for the relative discrepancy between this study and other similar studies (7,11-13). Although the current study was based on a sample size that was greater than in previous studies $(7,11,12)$ and yielded $97 \%$ statistical power, larg- er sample sizes from other populations and with clear indications for MSSO are still needed to validate the results of this study and to derive a highly valid 'evidence' determining the safest osteotomy design.

In conclusion, the location of MC and bone thickness in the region of MSSO were significantly variable according to age, but exhibited sexual diamorphism only in regions (B) and (C). This fundamental knowledge may help the maxillofacial surgeon to outline an osteotomy design tailored to the patient's age and sex. Nevertheless, the relative variability of the measurements between this study and other similar studies would stress the important rule of the preoperative analysis of the patient's own CBCT.

\section{References}

1. Wittwer G, Adeyemo WL, Beinemann J, Juergens P. Evaluation of risk of injury to the inferior alveolar nerve with classical sagittal split osteotomy technique and proposed alternative surgical techniques using computer-assisted surgery. Int J Oral Maxillofac Surg. 2012;41:79-86.

2. R. Trauner and H. Obwegeser. The surgical correction of mandibular prognathism and retrognathia with consideration of genioplastypart I: surgical procedures to correct mandibular prognathism and reshaping of the chin. Oral Surg Oral Med Oral Pathol. 1957;10:677-89. 3. Monson LA: Bilateral sagittal split osteotomy. Semin Plast Surg. 2013;27:145-8.

4. Verweij JP, Houppermans PN, Gooris P, Mensink G, van Merkesteyn JR. Risk factors for common complications associated with bilateral sagittal split osteotomy: A literature review and meta-analysis. J Craniomaxillofac Surg. 2016; 44:1170-80.

5. Panula K, Finne K, Oikarinen K. Incidence of complications and problems related to orthognathic surgery: a review of 655 patients. J Oral Maxillofac Surg. 2001;59:1128-36.

6. Cillo JE, Stella JP. Selection of sagittal split ramus osteotomy technique based on skeletal anatomy and planned distal segment movement: current therapy. J Oral Maxillofac Surg. 2005;63:109-14. 7. Scomparin L, Soares MQ, Rubira CM, Yaedú RY, Imada TS, Centurion BS, et al. CBCT location of the fusion between the buccal and lingual cortical in the mandibular ramus: importance to sagittal split osteotomy. Med Oral Patol Oral Cir Bucal. 2017;22:500-5.

8. Al-Shayyab MH. A simple method to locate mandibular foramen with cone-beam computed tomography and its relevance to oral and maxillofacial surgery: a radio-anatomical study. Surg Radiol Anat. 2018;40:625-34.

9. Bhardwaj D, Kumar JS, Mohan V. Radiographic evaluation of mandible to predict the gender and age. J Clin Diagn Res. 2014;8:ZC66-9. 10. Yu IH, Wong YK. Evaluation of mandibular anatomy related to sagittal split ramus osteotomy using 3-dimensional computed tomography scan images. Int J Oral Maxillofac Surg. 2008;37:521-8. 11. Sekerci AE, Sahman H. Cone beam computed tomographic analyses of the position and course of the mandibular canal: relevance to the sagittal split ramus osteotomy. Biomed Res Int. 2014;2014.

12. Huang CY, Liao YF. Anatomical position of the mandibular canal in relation to the buccal cortical bone in Chinese patients with different dentofacial relationships. J Formos Med Assoc. 2016;115:981-91. 13. Noleto JW, Marchiori E, Da Silveira HM. Evaluation of mandibular ramus morphology using computed tomography in patients with mandibular prognathism and retrognathia: relevance to the sagittal split ramus osteotomy. J Oral Maxillofac Surg. 2010;68:1788-94.

14. Tsuji Y, Muto T, Kawakami J, Takeda S. Computed tomographic analysis of the position and course of the mandibular canal: relevance to the sagittal split ramus osteotomy. Int J Oral Maxillofac Surg. 2005 34:243-6. 
15. Hur MS, Kim HC, Won SY, Hu KS, Song WC, Koh KS, et al. Topography and spatial fascicular arrangement of the human inferior alveolar nerve. Clin Implant Dent Relat Res. 2013;15:88-95.

16. Angelopoulos C, Thomas S, Hechler S, Parissis N, Hlavacek M. Comparison between digital panoramic radiography and cone-beam computed tomography for the identification of the mandibular canal as part of presurgical dental implant assessment. J Oral Maxillofac Surg. 2008;66:2130-5.

17. Hassan BA, Payam J, Juyanda B, Van der Stelt P, Wesselink PR. Influence of scan setting selections on root canal visibility with cone beam CT. Dentomaxillofac Radiol. 2012;41:645-8.

18. de Santana Santos T, Gomes AC, de Melo DG, Melo AR, Cavalcante JR, de Araújo LC, et al. Evaluation of reliability and reproducibility of linear measurements of cone-beam-computed tomography. Indian J Dent Res. 2012;23:473-8.

19. Sairam V, Geethamalika MV, Kumar PB, Naresh G, Raju GP. Determination of sexual dimorphism in humans by measurements of mandible on digital panoramic radiograph. Contemp Clin Dent. 2016;7:434-9.

20. Ashkenazi M, Taubman L, Gavish A. Age associated changes of the mandibular foramen position in anteroposterior dimension and of the mandibular angle in dry human mandibles. Anat Rec. 2011;294:1319-25.

21. Kanno CM, de Oliveira JA, Cannon M, Carvalho AA. The mandibular lingula's position in children as a reference to inferior alveolar nerve block. J Dent Child. 2005;72:56-60.

22. Aarabi M, Tabrizi R, Hekmat M, Shahidi S, Puzesh A. Relationship between mandibular anatomy and the occurrence of a bad split upon sagittal split osteotomy. J Oral Maxillofac Surg. 2014;72:250813.

23. Witherow H, Offord D, Eliahoo J, Stewart A. Postoperative fractures of the lingual plate after bilateral sagittal split osteotomies. $\mathrm{Br}$ J Oral Maxillofac Surg. 2006;44:296-300.

24. Chen JC, Lin LM, Geist JR, Chen JY, Chen CH, Chen YK. A retrospective comparison of the location and diameter of the inferior alveolar canal at the mental foramen and length of the anterior loop between American and Taiwanese cohorts using CBCT. Surg Radiol Anat. 2013;35:11-6.

\section{Conflict of interest}

The authors declare no conflict of interest. 